\title{
Influence of structural disorder on magnetic and transport properties of $\left(\mathrm{La}_{0.7} \mathrm{Sr}_{0.3}\right)_{0.5}\left(\mathrm{Pr}_{0.65} \mathrm{Ca}_{0.35}\right)_{0.5} \mathrm{MnO}_{3}$ films
}

\author{
V.G. Prokhorov, V.S. Flis, and G.G. Kaminsky \\ Institute of Metal Physics, National Academy of Sciences of Ukraine, Kiev, 03142, Ukraine \\ E-mail: pvg@imp.kiev.ua \\ Y.P. Lee and J.S. Park \\ Quantum Photonic Science Research Center and Department of Physics, Hanyang University \\ Seoul, 133-791 Korea \\ V.L. Svetchnikov \\ National Center of High-Resolution Electron Microscopy \\ Rotterdamseweg 137, 2628AL, Delft, The Netherlands
}

Received April 6, 2004, revised June 27, 2004

\begin{abstract}
Magnetic and transport properties of $\left(\mathrm{La}_{0.7} \mathrm{Sr}_{0.3}\right)_{0.5}\left(\mathrm{Pr}_{0.65} \mathrm{Ca}_{0.35}\right)_{0.5} \mathrm{MnO}_{3}$ films prepared by a «co-deposition» utilizing the laser-ablation technique are investigated in a wide temperature range. The film deposited at $300{ }^{\circ} \mathrm{C}$ has a nano-crystalline disordered structure and exhibits a paramagnetic temperature dependence of the magnetization with a narrow peak $(\Delta T \simeq 10 \mathrm{~K})$ at $T_{G} \simeq 45 \mathrm{~K}$, which can be interpreted as a paramagnetic $\rightarrow$ superparamagnetic transition. A short-term annealing of the as-deposited film at $750{ }^{\circ} \mathrm{C}$ leads to the formation of a high-textured polycrystalline microstructure and to the appearance of ferromagnetic (FM) and metal-insulator (MI) transitions at $T_{C} \simeq 240 \mathrm{~K}$ and $T_{P} \simeq 140 \mathrm{~K}$, respectively. The observed discrepancy between $T_{P}$ and $T_{C}$ values can be ascribed to a percolating nature of the MI transition, with an exponent of 5.3 for the percolating conductivity. The film deposited at $T_{\text {sub }} \simeq 740{ }^{\circ} \mathrm{C}$ is composed of the lattice strain-free and the lattice-strained crystallites with different lattice parameters and $T_{C}$ 's, and is consistently described in the framework of the Millis model [A.J. Millis, T. Darling, and A. Migliori, J. Appl. Phys. 83, 1588 (1998)]. For a single-phase crystalline film obtain $T_{C} \simeq 270 \mathrm{~K}$ and $T_{P} \simeq 260 \mathrm{~K}$.
\end{abstract}

PACS: 71.30.+h, 75.47.Gk, 75.47.Lx

\section{Introduction}

Half a century ago, Volger [1] found that a bulk sample of $\mathrm{La}_{0.8} \mathrm{Sr}_{0.2} \mathrm{MnO}_{3}$ exhibited a large magnetoresistance near room temperature. The recent discovery of colossal magnetoresistance (CMR) in thin films of the general formula $\mathrm{R}_{1-x} \mathrm{~A}_{x} \mathrm{MnO}_{3}$, where $\mathrm{R}$ is a rare-earth cation and $\mathrm{A}$ is alkali or alkaline earth cation [2,3], initiated numerous investigations not only because of their interesting fundamental science but because of possibilities for device applications $[4,5]$. Most of the early theoretical works on manganites focused on the relationship between the transport and magnetic properties and explained the coexistence of ferromagnetism and metallic behavior within the framework of the «double exchange» model, which considered the magnetic coupling between $\mathrm{Mn}^{3+}$ and $\mathrm{Mn}^{4+}$ ions, resulting from the motion of an electron between two partially filled $d$ shells governed by the strong on-site Hund's coupling [6-8]. In spite of considerable scientific efforts, the complex interplay of charge, lattice, spin, and orbital degrees of freedom in these systems is not completely understood. The situation is complicated significantly by the fact that the magnetic and the transport properties of the manganites are strongly dependent on the cation size, lattice strain, and microstructure.

As the CMR effect develops more strongly near the Curie point $\left(T_{C}\right)$, i.e., near the metal - insulator (MI) transition, which, in turn, reflects the electron-pho- 
non coupling and the antiferromagnetic superexchange and the FM exchange interactions, an analysis of the influence of the crystal structures on $T_{C}$ is very useful. A unified phase diagram as a function of the electronic transfer integral, which could be determined mainly by $\mathrm{Mn}-\mathrm{O}$ bond length and $\mathrm{Mn}-\mathrm{O}-\mathrm{Mn}$ angle, was presented recently [9]. The final result for $T_{C}$ can be written approximately in the following form $[10,11]$ :

$$
T_{C} \simeq x(1-x) W \sim \cos \varphi / d_{\mathrm{Mn}-\mathrm{O}}^{3.5},
$$

where $x$ is the concentration of a divalent ion, $W$ is the bandwidth, $\varphi$ is the tilt angle in the plane of the bond, and $d_{\mathrm{Mn}-\mathrm{O}}$ is the $\mathrm{Mn}-\mathrm{O}$ bond length. Therefore, any perturbation in the translation symmetry of the crystal lattice can lead to the variation of $\varphi$ and $d_{\mathrm{Mn}-\mathrm{O}}$ and, consequently, results in a change of $T_{C}$.

One way to control the stress exerted on the crystal lattice is a replacement of the rare-earth or the alkali ions by other ions with a different size. It is well known that $\operatorname{Pr}_{0.65} \mathrm{Ca}_{0.35} \mathrm{MnO}_{3}$ (PCMO), owing to a small Pr-ion radius, remains an insulator in both the paramagnetic and FM states [12], while the $\mathrm{La}_{0.7} \mathrm{Sr}_{0.3} \mathrm{MnO}_{3}$ (LSMO) shows a metallic behavior of the electrical resistance in the whole temperature range [13]. It was recently found that the substitution of the small-size $\mathrm{Pr}$ ion by $\mathrm{La}$ in $\mathrm{Pr}_{0.67} \mathrm{Ca}_{0.33} \mathrm{MnO}_{3}$ compound led to the appearance of a MI transition at low temperature owing to the melting of a charge-ordered insulating state [14]. On the other hand, the substitution of $\mathrm{Sr}$ for $\mathrm{Ca}$ in $\mathrm{Pr}_{0.7} \mathrm{Ca}_{0.3-x} \mathrm{Sr}_{x} \mathrm{MnO}_{3}$ induces the formation of the low-temperature metallic state, as well [15]. The influence of a lattice strain (and stress) accumulated during film deposition was intensively investigated, and it was shown that the lattice strain played an important role in the formation of spin- and charge-ordered states [16-19]. However, the influence of structurally quenched disorder on the magnetic ordering is still poorly understood.

In this paper we report our experimental results for $\left(\mathrm{La}_{0.7} \mathrm{Sr}_{0.3}\right)_{0.5}\left(\mathrm{Pr}_{0.65} \mathrm{Ca}_{0.35}\right)_{0.5} \mathrm{MnO}_{3}$ films prepared by a «co-deposition» utilizing the laser-ablation technique from two independent PCMO and LSMO targets. Several films with different structural order were prepared to investigate the influence of the different types of crystal disorder on the magnetic and transport properties of the films.

\section{Experimental techniques}

A cross-beam laser-ablation technique was employed for preparation of the films. A detailed description of the technique was presented elsewhere [20]. The deposition was carried out simultaneously from both
LSMO and PCMO targets. We used two Nd-YAG lasers with a wavelength of $1064 \mathrm{~nm}$, a pulse duration of 7.8-10.5 ns, a pulse-repetition rate of $20 \mathrm{~Hz}$, and an energy of $0.3 \mathrm{~J} /$ pulse. The power density of laser beam focused on the target was $9.5 \cdot 10^{8}-2 \cdot 10^{10} \mathrm{~W} / \mathrm{cm}^{2}$. The targets were prepared from the PCMO and LSMO powders of the stoichiometric composition by hotpressing and heating at $1200{ }^{\circ} \mathrm{C}$ for 4 days in air. The oxygen pressure in chamber was 200 Torr during deposition and 600 Torr during cooling. Under these conditions were grown $\left(\mathrm{La}_{0.7} \mathrm{Sr}_{0.3}\right)_{0.5}\left(\mathrm{Pr}_{0.65} \mathrm{Ca}_{0.35}\right)_{0.5} \mathrm{MnO}_{3}$ films at $T_{\text {sub }}=300$ (LPM1) and $740{ }^{\circ} \mathrm{C}$ (LPM2). In addition, the LPM1 and LPM2 films were annealed at $T_{\text {ann }}=750$ (LPM1A) and $900{ }^{\circ} \mathrm{C}$ (LPM2A) for $1 \mathrm{~h}$ in air, respectively. All the films were deposited on a $\mathrm{LaAlO}_{3}$ (001) single crystal and have a thickness of $d \simeq 200 \mathrm{~nm}$.

The $\theta-2 \theta$ x-ray diffraction (XRD) patterns were obtained using a Rigaku diffractometer with $\mathrm{Cu} K_{\alpha}$ radiation. The lattice parameters evaluated directly from the XRD data were plotted against $\cos ^{2} \theta / \sin \theta$. With an extrapolated straight line to $\cos ^{2} \theta / \sin \theta=0$, a more precise lattice parameter was obtained. The high-resolution electron-microscopy (HREM) studies were carried out by using a Philips CM300UT-FEG microscope with a field emission gun operated at $300 \mathrm{kV}$. The point resolution of the microscope was of the order of $0.12 \mathrm{~nm}$. The cross-sectional specimens were prepared by the standard techniques using mechanical polishing followed by ion-beam milling at a grazing incidence. The microstructure analysis was carried out at room temperature. The resistance measurements were performed by using the four-probe method in a temperature range of $4.2-300 \mathrm{~K}$ and in a magnetic field up to $5 \mathrm{~T}$. The in-plane field-cooled (FC) and zero-field-cooled (ZFC) magnetization was measured using a Quantum Design SQUID magnetometer in a temperature range of $4.2-300 \mathrm{~K}$.

\section{Experimental results}

\subsection{Microstructure of the films}

Figure 1, $a$ presents the (002) Bragg peaks for the LPM1 (curve 1) and the LPM1A (curve 2) films. It is seen that there is no crystalline phase in the LPM1 film and only the Bragg peak of the substrate is observable. It indicates the formation of an amorphous or a fine-crystalline disordered microstructure in the film. The LPM1A film displays only the high-intensity $(00 l)$ peaks, demonstrating that a short-term annealing at $750{ }^{\circ} \mathrm{C}$ results in a highly $c$-oriented film with an out-of-plane lattice parameter of $c \simeq 0.3855 \mathrm{~nm}$. The value obtained for the lattice parameter is in between that for the bulk LSMO $\left(a_{c}=0.3876 \mathrm{~nm}[13]\right)$ 


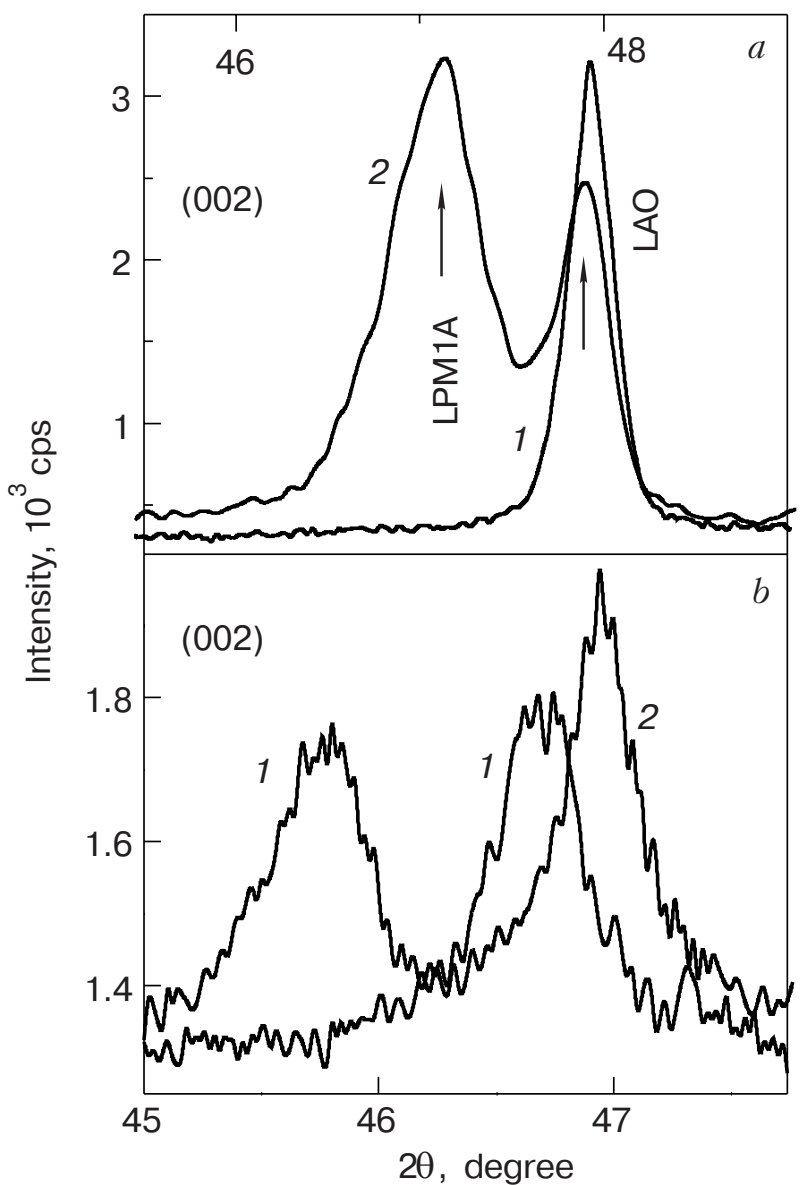

Fig. 1. (a) The (002) XRD peaks for the LPM1 (1) and the LPM1A (2) films. LAO denotes the substrate peaks. (b) The (002) XRD peaks for the LPM2 (1) and the LPM2A (2) films.

and PCMO $\left(a_{\mathrm{c}} \simeq 0.3843 \mathrm{~nm}[21]\right)$ compounds with a cubic symmetry. Figure $1, b$ displays that the LPM2 film manifests a split (002) Bragg peak (curve 1) and indicates the presence of two crystalline phases with $c \simeq 0.3961$ and $0.3888 \mathrm{~nm}$. An additional annealing at $900{ }^{\circ} \mathrm{C}$ results in a single-phase crystal structure (curve 2) in the film with $c \simeq 0.3873 \mathrm{~nm}$.

More-detailed information about the microstructure of the films can be obtained from the analysis of the HREM images. Figure 2 shows the cross-sectional HREM images for the LPM1 $(a)$ and the LPM1A $(b)$ films across the interface between film and substrate (indicated by white dashed lines). The electron-diffraction pattern, displayed in the inset of Fig. 2, $a$, demonstrates that the LPM1 film is not completely amorphous, as it has been recognized by the XRD data, but consists of a small-size $(D \simeq 5.5 \mathrm{~nm})$ disordered crystallites. Figure $2, b$ shows that annealing of the LPM1 film leads to the formation of a column-like high-textured microstructure with grain boundaries containing edge dislocations and regions of an uncrystallized phase. The inset of Fig. 2,b shows the fast

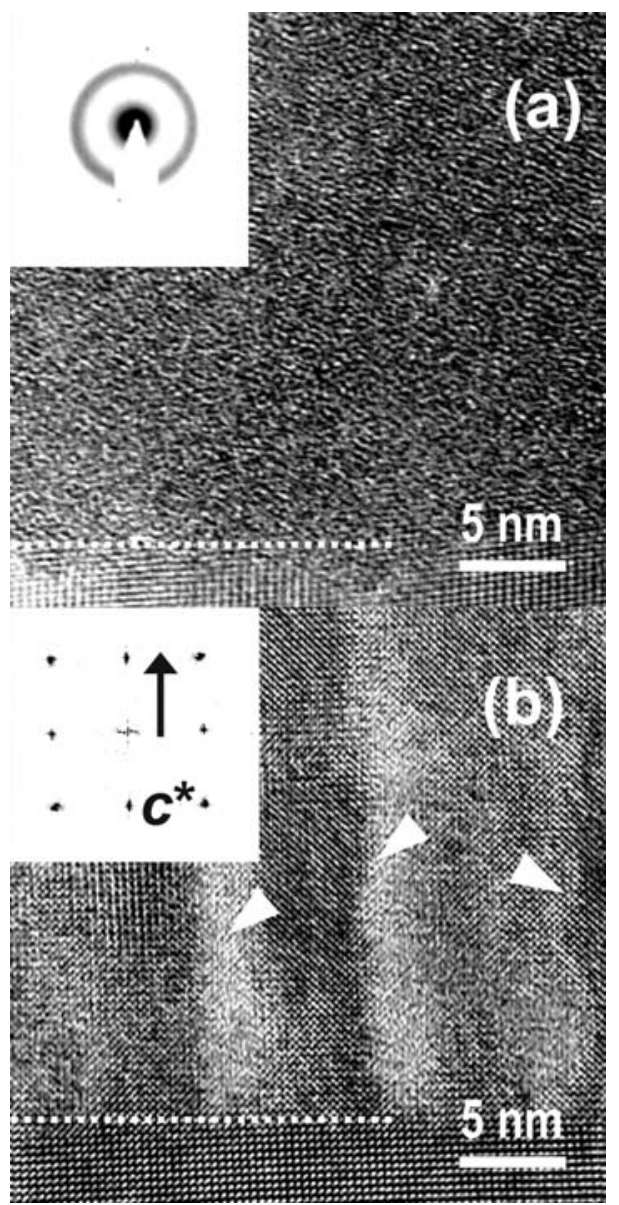

Fig. 2. Cross-sectional HREM images for LPM1 (a) and LPM1A ( $b$ ) films. The white arrows indicate the edge dislocations and the regions with uncrystallized phase. The inset in $(a)$ is the electron-diffraction pattern for LPM1, and that in $(b)$ is the FFT of the HREM image for LPM1A.

Fourier transform (FFT) of the HREM image for the LPM1A film. The FFT produces a rectangular pattern of almost circular spots. The measurement of a large number of interdot spacings allows us to obtain the average values of the lattice parameters from the HREM images. Upon the analysis, one can conclude that the LPM1A film has a pseudocubic crystal structure with $c \simeq a \simeq 0.386 \mathrm{~nm}$, which is in good agreement with the XRD data; here $a$ is the in-plane lattice parameter.

Figure 3 shows the cross-sectional HREM images for the LPM2 ( $a$ ) and the LPM2A ( $b$ ) films. The inset $A$ of Fig. 3, $a$ presents the FFT of the HREM image for the LPM2 film, displaying slightly split and elongated spots in both the $c$ (out-of-plane) and $a$ (in-plane) directions. These peculiarities of the FFT pattern indicate the presence of two crystalline phases with different lattice parameters. The inset $B$ of Fig. 3, $a$ shows a high-magnification HREM image of a small region of the LPM2 film. The measurement of 


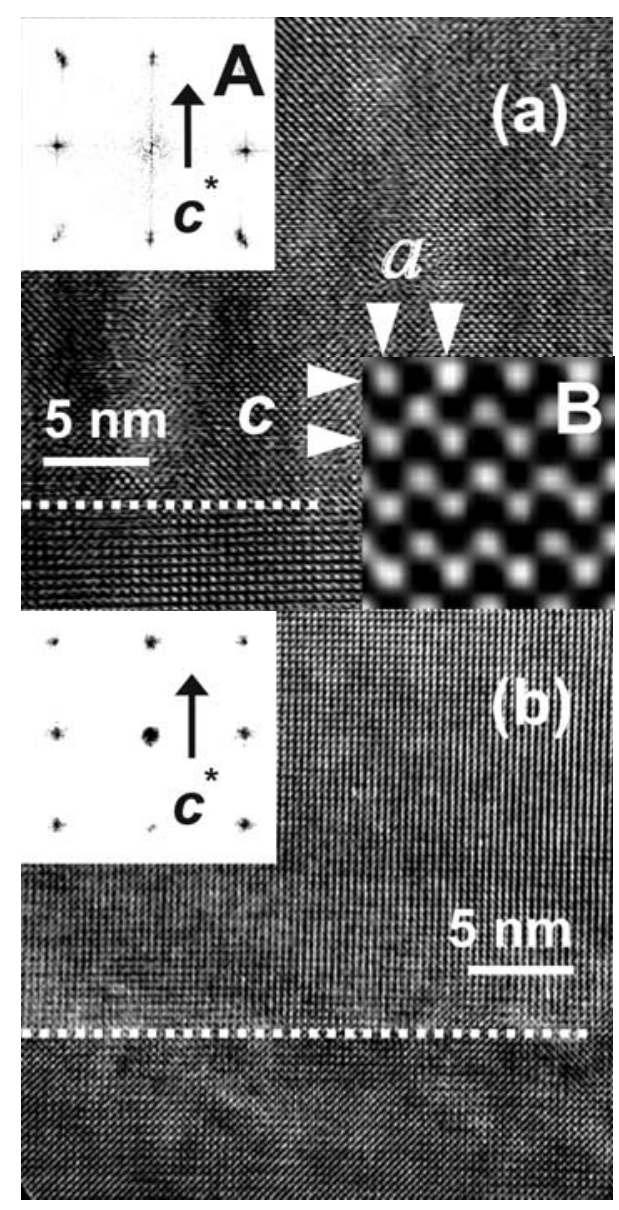

Fig. 3. Cross-sectional HREM images for LPM2 (a) and LPM2A (b) films. The inset $A$ is the FFT of HREM image and the inset $B$ is the high-magnification HREM image for LPM2. The inset in (b) is the FFT of HREM image for LPM2A.

a large number of interdot spacings for regions of this kind located in different areas of the film allows us to obtain the average values of the lattice parameters from the HREM images. Upon analysis, one can conclude that the LPM2 film consists of two crystalline phases with the following lattice parameters: $c \simeq a \simeq 0.388 \mathrm{~nm}$, and $c \simeq 0.396 \mathrm{~nm}$ and $a \simeq$ $\simeq 0.385 \mathrm{~nm}$, respectively. It is seen that the obtained results nicely coincide with the XRD data for the LPM2 film. We will show below that these two crystalline phases represent regions of the film with different levels of lattice strain. Nonuniformly distributed lattice strains of this kind have been observed in the CMR films [19].

Figure $3, b$ shows that an additional annealing leads to the removal of lattice strains in the LPM2 film and to the formation of a perfect crystal structure. The FFT, presented by the inset of Fig. 3,b, produces a rectangular pattern of circular and unsplit spots. A more detailed analysis of the HREM images of
LPM2A shows that the film has a pseudocubic crystal structure with lattice parameters $c \simeq a \simeq 0.387 \mathrm{~nm}$. Therefore, $\left(\mathrm{La}_{0.7} \mathrm{Sr}_{0.3}\right)_{0.5}\left(\mathrm{Pr}_{0.65} \mathrm{Ca}_{0.35}\right)_{0.5} \mathrm{MnO}_{3}$ films with different kinds of crystal structure were prepared, and the magnetic and the transport properties were investigated: nano-scale disordered (LPM1), column-like polycrystalline (LPM1A), nonuniformly strained (LPM2), and single-crystalline strain-free (LPM2A) films.

\subsection{Magnetic and transport properties}

Figure 4 presents both the FC (filled dots) and ZFC (unfilled dots) temperature-dependent magnetization curves for the LPM1 $(a)$, the LPM1A $(b)$, the LPM2 $(c)$, and the LPM2A (d) films. The LPM1 film deposited at low substrate temperature shows a typical paramagnetic $M(T)$ dependence with a neglible splitting between the FC and ZFC curves in a magnetic field of 500 Oe and with a rapid growth of $M$ as $T \rightarrow 0$. On the other hand, a narrow peak in the magnetization was found at $T_{G} \simeq 45 \mathrm{~K}$. A slight negative contribution to the magnetization of the film is provided by the substrate owing to its diamagnetism, with an estimated value of the susceptibility $\chi_{\text {dia }} \simeq-5.6 \cdot 10^{-4} \mathrm{~cm}^{3} / \mathrm{g}$ for our case. Figure $4, b$ shows that annealing of the film at $740{ }^{\circ} \mathrm{C}$ leads to the formation of a FM state with $T_{C} \simeq 240 \mathrm{~K}$. The LPM2 film exhibits the $M(T)$ dependence typical for two-phase magnetic systems (see Fig. 4,c) and represents a superposition of two magnetic transitions at $T_{C 1} \simeq 270 \mathrm{~K}$ and $T_{C 2} \simeq 180 \mathrm{~K}$.

It is worth noting that both transition temperatures are significantly different from those for bare PCMO $\left(T_{N} \simeq 130 \mathrm{~K}\right)[20]$ and $\operatorname{LSMO}\left(T_{C} \simeq 340 \mathrm{~K}\right)$ films [16]. The results are coincident with the XRD and

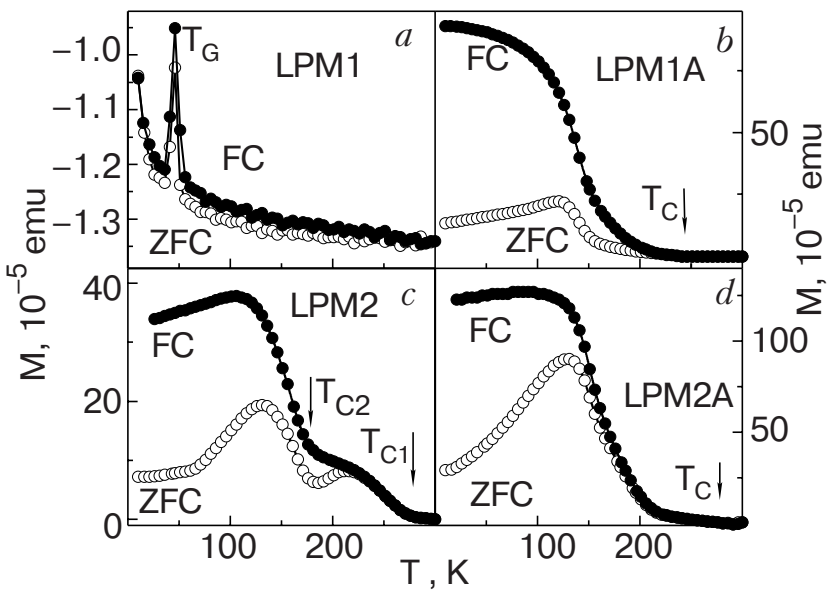

Fig. 4. The FC (filled dots) and ZFC (unfilled dots) magnetization curves for the LPM1 $(a)$, the LPM1A $(b)$, the LPM2 $(c)$, and the LPM2A $(d)$ films. The solid lines are a guide for the eye. 


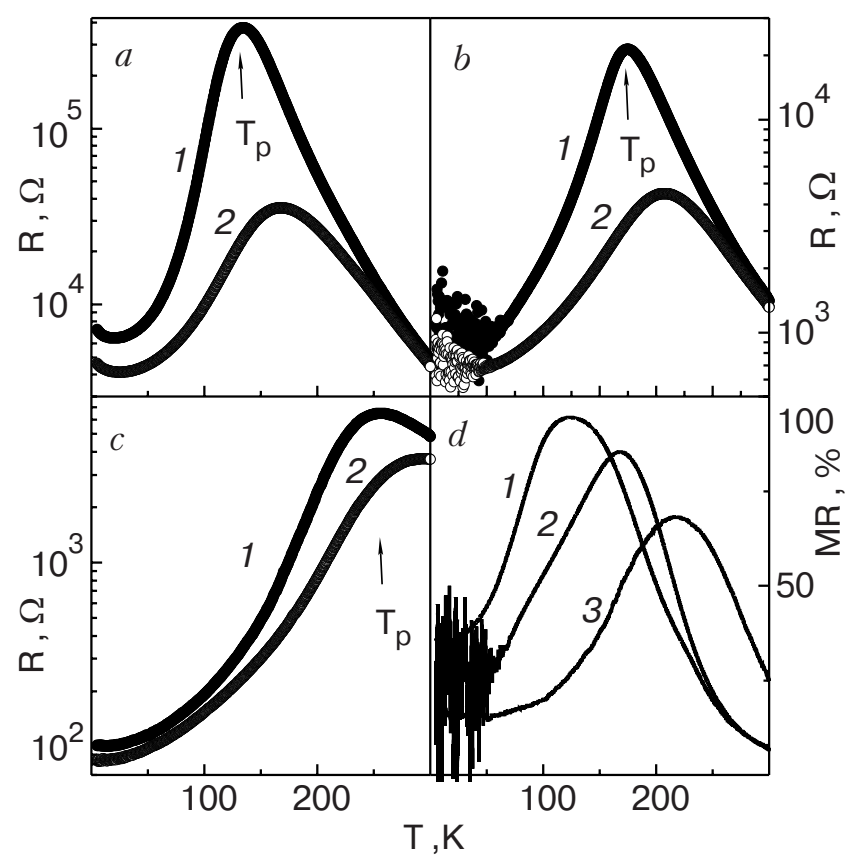

Fig. 5. The temperature-dependent resistance without (1) and with (2) an applied magnetic field of $5 \mathrm{~T}$ for the LPM1A ( $a)$, the LPM2 (b) and the LPM2A (c) films. (d) The MR ratio for the LPM1A (1), the LPM2 (2) and the LPM2A (3) films in an applied magnetic field of $5 \mathrm{~T}$.

HREM data, and confirm the presence of two different crystalline phases in the film. Annealing at $900{ }^{\circ} \mathrm{C}$ leads to homogenization of these phases and to the formation of the FM state in the whole film with $T_{C} \simeq 270 \mathrm{~K}$. Figure $4, d$ shows that the value of the spontaneous magnetization for the LPM2A film is significantly larger than that for the LPM2 film.

Unfortunately, we could not measure $R(T)$ for the LPM1 film, since our setting was limited to $10^{7} \Omega$ and the resistance was larger than $10^{7} \Omega$ at room temperature. Figure 5 displays $R(T)$ for the LPM1A $(a)$, LPM2 (b), and LPM2A (c) films without (1) and with (2) an applied magnetic field of $5 \mathrm{~T}$. The magnetic field was directed perpendicular to the transport current and parallel to the film surface. Figure 5, $a$ demonstrates that the MI transition in the LPM1A film occurs at $T_{P} \simeq 140 \mathrm{~K}$, which is far below $T_{C}$ for this film (see Fig. 4,b). $R(T)$ displays a resistance minimum at low temperature, which is very often observed in polycrystalline CMR films $[22,23]$. The position of the resistance peak for the LPM2 film is shifted toward higher temperature, $T_{P} \simeq 180 \mathrm{~K}$, and is equal to the temperature of the second magnetic transition in this film, $T_{C 2} \simeq 180 \mathrm{~K}$ (see Fig. 4,c).

Figure 5,b shows that the random resistance oscillations appear in the $R(T)$ curve in a low-temperature range, which are slightly suppressed under the applied magnetic field of $5 \mathrm{~T}$. Annealing of the LPM2 film at $900{ }^{\circ} \mathrm{C}$, as is demonstrated in the Fig. 5,c, leads to an increase of $T_{P} \simeq 260 \mathrm{~K}$ and to the disappearance of the resistance oscillations at low temperature. Figure $5, d$ exhibits the temperature dependence of the negative magnetoresistance (MR) for the LPM1A (1), LPM2 (2), and LPM2A (3). The MR value is defined as $100 \% \cdot[R(0)-R(H)] / R(0)$, where $R(0)$ and $R(H)$ are the resistances with and without a magnetic field of $5 \mathrm{~T}$, respectively. It is seen that the increase of $T_{P}$ is accompanied by a decreasing MR effect, which is typical for the CMR compounds [4,5].

\section{Discussion}

Let us first consider the magnetic behavior of the nano-crystalline disordered LPM1 film. Figure 6 shows the in-plane FC $M(T)$ dependence measured under an applied magnetic field of 500 Oe. It is seen that the observed narrow peak at $T_{G} \simeq 45 \mathrm{~K}$ divides the $M(T)$ curve into two temperature ranges with different $M(T)$ behavior. Below $T_{G}$ the $M(T)$ curve can be described in the framework of the Curie-Weiss (CW) approximation [24]

$$
M^{P M}(T, H)=\left(\chi_{0}+\frac{C_{C W}}{T+\theta}\right) H,
$$

where $\chi_{0}$ is a temperature-independent susceptibility, and the second term is the CW-type susceptibility with a constant $C_{C W}$ and a characteristic temperature $\theta$. Figure 6 demonstrates that the experimental data for LPM1 can be excellently described by the $\mathrm{CW}$ expression with following parameters: $\chi_{0} \simeq$ $\simeq 3.3 \cdot 10^{-4} \mathrm{~cm}^{3} / \mathrm{g}, \quad C_{C W} \simeq 1.01 \cdot 10^{-2} \mathrm{~cm}^{3} / \mathrm{g}, \quad$ and $\theta=5 \mathrm{~K}$. The effective moment estimated from $C_{C W}$ turns out to be $\mu_{\text {eff }} \simeq 4.2 \mu_{B} / \mathrm{Mn}$, which is almost coincident with the theoretical value, $\mu_{\text {eff }}^{\text {theor }} \simeq$ $\simeq 4.6 \mu_{B} / \mathrm{Mn}$, obtained from following expression [24]:

$$
\begin{aligned}
& \mu_{\mathrm{eff}}^{\text {theor }}=g\left\{0 . 5 \left[x S_{1}\left(S_{1}+1\right)+(1-x) s_{2}\left(S_{2}+1\right)+\right.\right. \\
& \left.\left.+y S_{1}\left(S_{1}+1\right)+(1-y) S_{2}\left(S_{2}+1\right)\right]\right\}^{1 / 2} .
\end{aligned}
$$

Here $x$ and $y$ are the $\mathrm{Ca}$ and the Sr concentration, $S_{1}=3 / 2$ and $S_{2}=2$ are the spin values of the $\mathrm{Mn}^{4+}$ and $\mathrm{Mn}^{3+}$ ions, respectively, and $g=2$ is the Lande factor. Therefore, one can conclude that the nanocrystalline disordered LPM1 film is a typical paramagnet in the temperature range below $T_{G}$, with a free motion of the individual Mn spins. On the other hand, the magnetization decreases sharply and deviates from the $\mathrm{CW}$-type straight line at $T \geq T_{G}$. Such nonlinear behavior of $M\left(T^{-1}\right)$ is rather typical for superparamagnetic (SPM) particles and can be described by a Langevin function as [24]

$$
M^{S P M}(T, H)=M_{S}^{S P M}\left[\operatorname{coth}\left(\frac{\mu H}{k_{B} T}\right)-\frac{k_{B} T}{\mu H}\right],
$$




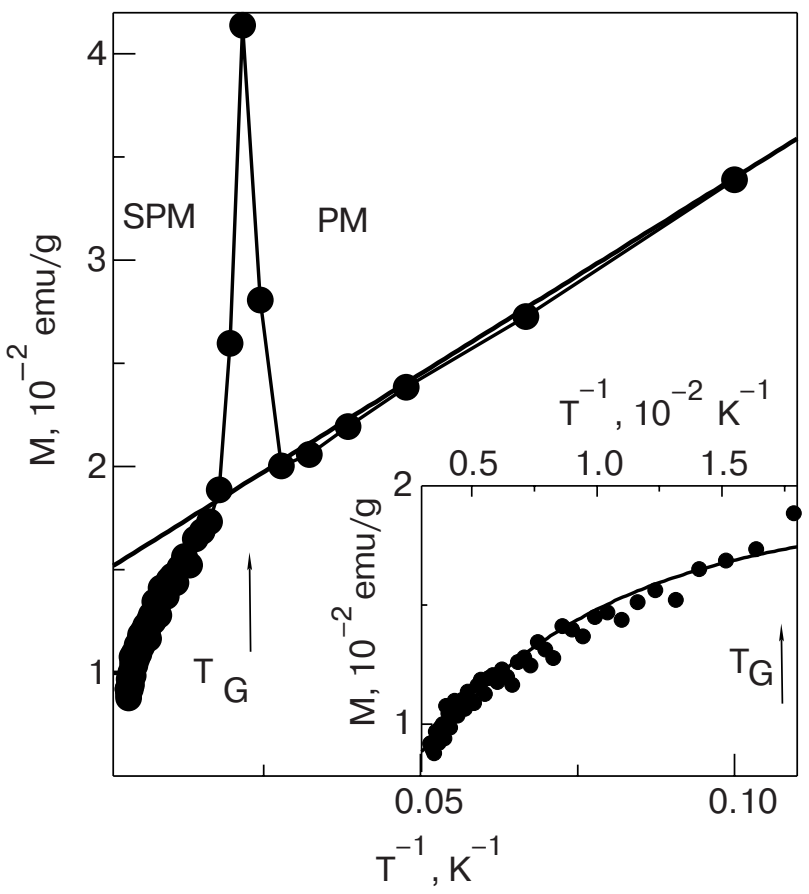

Fig. 6. $M$ versus $T^{-1}$ plot for the LPM1 film under an applied field of $H=100$ Oe. The solid line represents the CW-type paramagnetic approximation. The inset displays the same plot above $T \geq T_{G}$. The solid line represents a Langevin function describing the magnetic behavior of SPM particles.

where $M_{S}^{S P M}$ is the saturation magnetization of the SPM phase and $\mu$ is the average magnetic moment of the SPM particles. The solid line of the inset of Fig. 6 represents a Langevin function giving the best fit to the experimental data above $T_{G}$. This line corresponds to the magnetization contribution of SPM particles having an average moment $\mu \simeq 7500 \mu_{B}$. By taking 3.4 $\mu_{B} / \mathrm{Mn}$ atom [25] and assuming a spherical shape of the SPM clusters, with a volume of $\pi D^{3} / 6$, we estimated their average diameter as $D \simeq$ $\simeq 6 \mathrm{~nm}$. Because the estimated diameter is very close to the crystallite size revealed by the electron diffraction, one can conclude that these nano-scale disordered crystallites play the role of the superparamagnetic particles. The narrow peak found in the $M\left(T^{-1}\right)$ curve manifests a phase transition from SPM to PM state, and $T_{G}$ can be interpreted as the temperature for spin-glass (or cluster-glass) freezing.

Figure $4, b$ shows that the annealing of the nano-crystalline disordered LPM1 film leads to its recrystallization and to a recovery of FM state. On the other hand, the large difference between ZFC and FC magnetization curves and a significant discrepancy between the MI and FM transition temperatures manifest the coexistence of the FM metallic and the PM insulating clusters below $T_{C}[26,27]$. A similar me- tastable-phase mixture was observed recently in the parent $\left(\mathrm{La}_{1-x} \mathrm{Pr}_{x}\right)_{0.7} \mathrm{Ca}_{0.3} \mathrm{MnO}_{3}$ compound and explained by the phase separation effect $[14,25]$. However, in our case the phase-mixed state has a metallurgical rather than an electronic nature and is related to the presence of the dislocation networks in the LPM1A film and the regions of an uncrystallized phase. Therefore, the MI transition cannot be treated as a real electronic one but is governed by the percolation of the FM metallic domains [28].

According to the percolation theory, the conductivity can be expressed as $\sigma \sim\left(p-p_{0}\right)^{t}$, where $p$ is the concentration of the metallic phase, $p_{0}$ is its threshold value in the vicinity of $0.4-0.5$ [28], where metallic filaments are created that permit current transmission across the sample, and $t$ is the exponent. Figure 7 shows the temperature dependence for both the normalized resistance $r=R(T) / R\left(T_{P}\right)$ and spontaneous magnetization $m=M(T) / M(0)$ for the LPM1A film. It is seen that the temperature location of the resistance peak, $T_{P}$, corresponds to $m \simeq 0.4$, which is coincident with the percolating threshold value and allows us to use the magnetization as a percolating parameter for the conducting phase. The inset $(b)$ of Fig. 7 represents the $\lg (1 / r)$ versus $\lg \left(m-m_{0}\right)$ plot, where $m_{0}=0.4$. The solid lines are the fitted curves with fol-

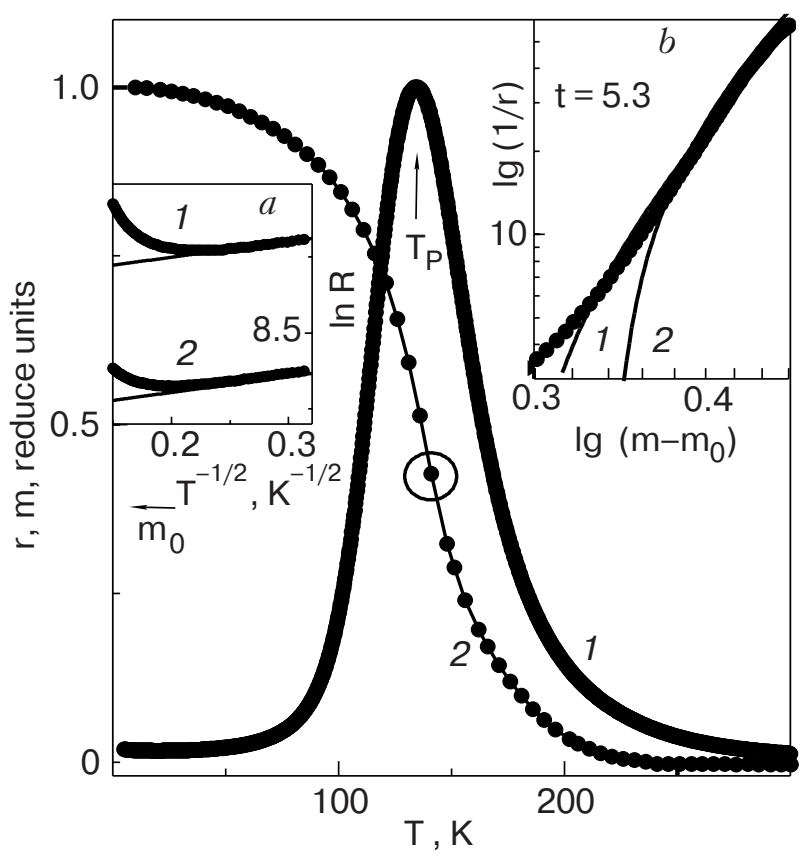

Fig. 7. $r(T)$ and $m(T)$ for the LPM1A film. The circle on the $m(T)$ indicates the critical volume of the FM phase $\left(m_{0}\right)$ for the percolating transition. The inset $(a)$ displays the $\ln R$ versus $T^{-1 / 2}$ plot for the LCP1A film without (1) and with (2) an applied magnetic field of $5 \mathrm{~T}$. The inset $(b)$ displays the $\lg (1 / r)$ versus $\lg \left(m-m_{0}\right)$ plot. Solid lines correspond to the fitting with the percolating exponents $t \simeq 5.3$ (1) and $\simeq 3.6$ (2), respectively. 
lowing exponents for the percolating conductivity: $t \simeq 5.3$ (curve 1) and 3.6 (curve 2). Although the value $t \simeq 3.6$ was obtained recently from an analysis of the MI transition at high magnetic field for $\mathrm{Pr}_{0.63} \mathrm{Ca}_{0.37} \mathrm{MnO}_{3}$ [29], in our case $t \simeq 5.3$ is more appropriate for the description of the experimental curve. This value of $t$ is very close to that obtained by a numerical calculation for a 3-dimensional system with spin effects [30]. Therefore, one can conclude that the MI transition in the LPM1A film has a percolating nature.

The inset $(a)$ of Fig. 7 displays the $\ln R$ versus $T^{-1 / 2}$ plot for the LPM1A film without (curve 1) and with (2) an applied magnetic field of $5 \mathrm{~T}$. This plot manifests the exponential growth of resistance at low temperature, which is described by an expression: $R(T) \sim \exp \sqrt{\Delta / T}$. It noteworthy that a similar expression with an energy gap $\Delta \sim E_{C}$, where $E_{C}$ is the charging energy (or Coulomb barrier), has been predicted for the conductivity in granular metals [31] and used to explain the low-temperature $R(T)$ behavior in ceramic $\mathrm{La}_{2 / 3} \mathrm{Sr}_{1 / 3} \mathrm{MnO}_{3}$ manganite [32]. Because the crystalline phase in LPM1A was grown from the nano-crystalline disordered phase under equilibrium thermodynamic conditions, the GBs of this film contain a higher concentration of edge dislocations and segregated impurities than those usually observed in epitaxially grown films, and this results in the formation of an additional small Coulomb barrier. The value of the charging energy estimated from the slope of the ln $R$ versus $T^{-1 / 2}$ plot is $E_{C} \simeq 0.0233 \mathrm{meV}$ for both cases (without and with an applied magnetic field).

The fact that the value obtained for the charging energy of the LPM1A film is significantly smaller than that for ceramic manganites [32] attests to a better electronic transparency of GBs in this film. On the other hand, the insensitivity of $E_{C}$ to the applied magnetic field proves that just the Coulomb barrier is formed in GBs.

The magnetization data show that the as-deposited LPM2 film demonstrates the temperature behavior typical for two-phase systems (see Fig. 4,c). It is also confirmed by the XRD and the HREM data. However, a short-term annealing at $900{ }^{\circ} \mathrm{C}$ leads to formation of a single-phase crystal structure (see Figs. $1, b$ and $3, b)$. Our recent investigations of $\mathrm{Pr}_{0.65} \mathrm{Ca}_{0.35} \mathrm{MnO}_{3}$ films show that annealing at $900{ }^{\circ} \mathrm{C}$ for up to 10 hours does not change the chemical composition of the film (including the oxygen content) but leads only to relaxation of the lattice strains [20].

Therefore, one can suggest that the main difference between the LPM2 and the LPM2A films is a different concentration of the lattice strains only. It is believed that, owing to a significant lattice mismatch between the substrate and the film, lattice strains are accumulated in the film during deposition. As reported recently, under a compressive biaxial strain the film grows in the islands mode, and the strains are distributed nonuniformly through the sample [19]. The edge of an island is a region of high strain, while the top of an island is a region of low strain. Consequently, the LPM2 film represents a composition of compressive biaxial strain $(c \simeq 0.396 \mathrm{~nm}$ and $a \simeq 0.385 \mathrm{~nm})$ and strain-free $(c \simeq a \simeq 0.388 \mathrm{~nm})$ crystallites. This is also confirmed by the fact that the lattice parameters of the strain-free regions remain practically unchanged after annealing $(c \simeq a \simeq 0.387 \mathrm{~nm})$. Therefore, the observed two-phase $M(T)$ behavior of LPM2 (see Fig. 4,c) can be explained by the existence of these differently strained crystallites. Let us prove this conjecture to be true on the basis of modern theoretical approaches. For weaker strains and a cubic symmetry $T_{C}$ can be expressed, according to the Millis model, by [33]:

$$
T_{C}(\varepsilon)=T_{C}(\varepsilon=0)\left(1-\alpha \varepsilon_{B}-\frac{1}{2} \Delta \varepsilon_{J T}^{2}\right),
$$

where $\varepsilon_{B}=\left(2 \varepsilon_{100}+\varepsilon_{001}\right)$ is a bulk strain, $\varepsilon_{100}=$ $\left(a_{\mathrm{film}}-a_{\mathrm{bulk}}\right) / a_{\text {bulk }}$ is the in-plane biaxial compressive strain, $\varepsilon_{001}=\left(c_{\text {film }}-c_{\text {bulk }}\right) / c_{\text {bulk }}$ is the outof-plane uniaxial tensile strain, $a_{\mathrm{film}}, a_{\mathrm{bulk}}, c_{\mathrm{film}}$, and $c_{\text {bulk }}$ are the in-plane and out-of-plane lattice parameters for the film and the bulk, respectively, $\varepsilon_{J T}=$ $=\sqrt{2 / 3}\left(\varepsilon_{001}-\varepsilon_{100}\right)$ is the Jahn - Teller strain, $\alpha=$ $=\left(1 / T_{C}\right)\left(d T_{C} / d \varepsilon_{B}\right)$, and $\Delta=\left(1 / T_{C}\right)\left(d^{2} T_{C} / d \varepsilon_{J T}^{2}\right)$. The magnitudes of $\alpha$ and $\Delta$ represent the relative weights for the symmetry-conserving bulk and the symmetry-breaking Jahn - Teller strains, respectively. According to the theoretical model [33], $\alpha \simeq 10$ for a reasonable electron-phonon coupling $(0.5 \leq \lambda \leq 1)$ in these compounds, where $\lambda$ is the electron-phonon interaction constant. If only the relative difference in the lattice strain between the strain-free and the strained crystallites is considered, the strain-free phase can be treated as a bulk with $c_{\text {bulk }} \simeq a_{\text {bulk }} \simeq$ $\simeq 0.388 \mathrm{~nm}$. The lattice parameters estimated from the HREM image (Fig. 3,a) are used for the strained phase. Using $T_{C}$ for the strain-free phase as $T_{C 1}=T_{C}(\varepsilon=0) \simeq 270 \mathrm{~K}$ and the obtained values of $\varepsilon_{100}$ and $\varepsilon_{001}$ and $T_{C 2} \simeq 180 \mathrm{~K}$ for the strained phase in the LPM2 film, we can obtain $\Delta \simeq 1500$, which is almost coincident with the prediction of a theoretical model [33]. Therefore, one can conclude that the observed magnetic phase separation in the as-deposited LPM2 film is governed by the nonuniform distribution of the lattice strains. 
A comparison of the $M(T)$ and $R(T)$ curves for the LPM2 film (see Figs. 4,c and 5,b) demonstrates that the MI transition occurs at a temperature $T_{P} \simeq 180 \mathrm{~K}$, which is coincident with $T_{C}$ for a lattice strained phase, $T_{C 2} \simeq 180 \mathrm{~K}$. This can be explained by a large difference in their volumes in the film, which is confirmed by the sharp increase in the magnetization (almost four times) at $T \leq T_{C 2}$. Therefore, the concentration of the FM metallic phase, which appears at the first magnetic transition of $T_{C 1} \simeq 270 \mathrm{~K}$, is insufficient for the formation of an infinite percolating network in the film, and only a single MI transition is observed on the $R(T)$ dependence.

The observed random oscillations of resistance in the LPM2 film at low temperature are probably due to the presence of the lattice strain, because this effect disappears after annealing (see Fig. 5). Unfortunately, we did not carry out a detailed investigation of this phenomenon in this work, but it is worth noting that a similar effect has recently been observed by us in the nano-crystalline twinned $\mathrm{La}_{0.65} \mathrm{Ca}_{0.35} \mathrm{MnO}_{3}$ films [34].

Figures 4, $d$ and 5,c demonstrate that the annealed LPM2A film undergoes the FM and the MI transitions at $T_{C} \simeq 270 \mathrm{~K}$ and $T_{P} \simeq 260 \mathrm{~K}$, respectively. The magnetic and transport characteristics for this $\left(\mathrm{La}_{0.7} \mathrm{Sr}_{0.3}\right)_{0.5}\left(\mathrm{Pr}_{0.65} \mathrm{Ca}_{0.35}\right)_{0.5} \mathrm{MnO}_{3}$ film are significantly better than those observed for the $\mathrm{La}_{0.4} \mathrm{Pr}_{0.27} \mathrm{Ca}_{0.33} \mathrm{MnO}_{3}$ film deposited on LAO [14], although in our case the concentration of $\mathrm{Pr}$ is slightly larger (La:Pr is $0.52: 0.48$ ). We may argue that observed enhancement of the FM and MI transition temperatures is closely related to the substitution of the $\mathrm{Ca}$ ions by the $\mathrm{Sr}$ ones. A similar effect was observed recently in ceramic $\operatorname{Pr}_{0.65}\left(\mathrm{Ca}_{y} \mathrm{Sr}_{1-y}\right)_{0.35} \mathrm{MnO}_{3}$ compounds [35]. Therefore, one can conclude that the $\mathrm{Sr}$ ions play a more positive role in these compounds, leading to an increase of $T_{C}$, than the Pr ions, which should suppress the FM ordering.

\section{Conclusions}

$\left(\mathrm{La}_{0.7} \mathrm{Sr}_{0.3}\right)_{0.5}\left(\mathrm{Pr}_{0.65} \mathrm{Ca}_{0.35}\right)_{0.5} \mathrm{MnO}_{3}$ films with different crystal structure have been prepared by a «co-deposition» utilizing the laser-ablation technique from two independent PCMO and LSMO targets. XRD and HREM analysis reveal that the film deposited at a substrate temperature of $300{ }^{\circ} \mathrm{C}$ has a nano-crystalline disordered structure and does not undergo the FM transition in the whole temperature range. A narrow peak $(\Delta T \simeq 10 \mathrm{~K})$ in a temperature-dependent magnetization was observed at $T_{G} \simeq 45 \mathrm{~K}$, which was interpreted as a $\mathrm{PM} \rightarrow \mathrm{SPM}$ transition. It was shown that the nano-scale crystallites play a role of the superparamagnetic clusters in the film. The annealing at $750{ }^{\circ} \mathrm{C}$ for $1 \mathrm{~h}$ in air leads to a recrystallization of the film and to the appearance of the FM and MI transitions at $T_{C} \simeq 240 \mathrm{~K}$ and $T_{P} \simeq 140 \mathrm{~K}$, respectively. The observed discrepancy between $T_{P}$ and $T_{C}$ values is explained by a percolating nature of the MI transition. The film deposited at $T_{\text {sub }} \simeq 740{ }^{\circ} \mathrm{C}$ is composed of the lattice strain-free and the lattice-strained crystallites with different lattice parameters and $T_{C}$ 's. The strain-driven change in $T_{C}$ was consistently described on the basis of the Millis model [33]. The annealing at $900{ }^{\circ} \mathrm{C}$ leads to the formation of a single-phase crystal structure with $T_{C} \simeq 270 \mathrm{~K}$ and $T_{P} \simeq 260 \mathrm{~K}$.

This work was supported by the KOSEF through the Quantum Photonic Science Research Center.

1. J. Volger, Physica (Utrecht) 20, 49 (1954).

2. R. von Helmolt, J. Wecker, B. Holzapfel, L. Schultz, and K. Samwer, Phys. Rev. Lett. 71, 2331 (1993).

3. S. Jin, T.H. Tiefel, M. McCormack, R.A. Fastnacht, R. Ramesh, and L.H. Chen, Science 264, 413 (1994).

4. Y. Tokura and Y. Tomioka, J. Magn. Magn. Mater. 200, 1 (1999).

5. A.P. Raminez, J. Phys. C9, 8171 (1997).

6. C. Zener, Phys. Rev. 82, 403 (1951).

7. P.W. Anderson and H. Hasegawa, Phys. Rev. 100, 675 (1955).

8. P.-G. de Gennes, Phys. Rev. 118, 141 (1960).

9. H.Y. Hwang, S.W. Cheong, N.P. Ong, and B. Batlogg, Phys. Rev. Lett. 77, 2041 (1996).

10. C.M. Varma, Phys. Rev. B54, 7328 (1996).

11. M. Medarde, M. Mesot, P. Lacorre, S. Rosenkranz, P. Fisher, and K. Grobcht, Phys. Rev. B52, 9248 (1995).

12. Y. Tomioka, A. Asamitsu, H. Kuwahara, Y. Morimoto, and Y. Tokura, Phys. Rev. B53, R1689 (1996).

13. M.C. Martin, G. Shirane, Y. Endoh, K. Hirota, Y. Morimoto, and Y. Tokura, Phys. Rev. B53, 14285 (1996).

14. T. Wu, S.B. Ogale, S.R. Shinde, A. Biswas, T. Polletto, R.L. Greene, T. Venkatesan, and A.J. Millis, J. Appl. Phys. 93, 5507 (2003).

15. I. Medvedeva, A. Maignan, K. Bärner, Yu. Bersenev, A. Roev, and B. Reveau, Physica B325, 57 (2003).

16. F. Tsui, M.C. Smoak, T.K. Nath, and C.B. Eom, Appl. Phys. Lett. 76, 2421 (2000).

17. R.A. Rao, D. Lavric, T.K. Nath, C.B. Eom, L. Wu, and F. Tsui, Appl. Phys. Lett. 73, 3294 (1998).

18. S. Jacob, T. Roch, F.S. Razavi, G.M. Gross, and H.-U. Habermeier, J. Appl. Phys. 91, 2232 (2002).

19. A. Biswas, M. Rajeswari, R.C. Srivastava, T. Venkatesan, R.L. Green, Q. Lu, A.L. de Lozanne, and A.J. Millis, Phys. Rev. B63, 184424 (2001).

20. V.G. Prokhorov, G.G. Kaminsky, V.S. Flis, Y.P. Lee, K.W. Kim, and I.I. Kravchenko, Physica B307, 239 (2001).

21. Z. Jirák, S. Krupička, Z. Šimvša, M. Dlouhá, and S. Vratislav, J. Magn. Magn. Mater. 53, 153 (1985). 
22. R. Gross, L. Alff, B. Büchner, B.H. Freitag, C. Höfener, J. Klein, Y. Lu, W. Mader, J.B. Philipp, M.S. R. Rao, P. Reutler, S. Ritter, S. Thienhaus, S. Uhlenbruck, and B. Wiedenhorst, J. Magn. Magn. Mater. 211, 150 (2000).

23. M. Auslender, A.E. Karkin, E. Rozenberg, and G. Gorodetsky, J. Appl. Phys. 89, 6639 (2001).

24. B.D. Cullity, Introduction to Magnetic Materials, Addison-Wesley, New York (1972).

25. A.M. Balagurov, V.Yu. Pomjakushin, D.V. Sheptyakov, V.L. Aksenov, P. Fischer, L. Keller, O.Yu. Gorbenko, A.R. Kaul, and N.A. Babushkina, Phys. Rev. B63, 024420 (2001).

26. C.S. Hong, W.S. Kim, and N.H. Hur, Phys. Rev. B63, 092504 (2001).

27. M. Föth, S. Freisem, A.A. Menovsky, Y. Momioka, J. Aarts, and J.A. Mydosh, Science 285, 1540 (1999).

28. E. Dagotto, T. Hotta, and A. Moreo, Phys. Rep. 344, 1 (2001).
29. V. Hardy, A. Wahl, and C. Martin, Phys. Rev. B64, 064402 (2001).

30. Y. Xiong, S.-Q. Shen, and X.C. Xie, Phys. Rev. B63, 140418 (2001)

31. P. Sheng, B. Abeles, and Y. Arie, Phys. Rev. Lett. 31, 44 (1973).

32. Ll. Balcells, J. Fontcuberta, B. Martínez, and X. Obradors, Phys. Rev. B58, R14 697 (1998).

33. A.J. Millis, T. Darling, and A. Migliori, J. Appl. Phys. 83, 1588 (1998).

34. V.G. Prokhorov, G.G. Kaminsky, V.A. Komashko, Y.P. Lee, A.I. Tovstolytkin, and A.N. Pogorily, Fiz. Nizk. Temp. 28, 1199 (2002) [Low Temp. Phys. 28, 856 (2002)].

35. G.R. Blake, L. Chapon, P.G. Radaelli, D.N. Argyriou, M.J. Gutmann, and J.F. Mitchell, Phys. Rev. B66, 144412 (2002). 